\title{
On the Propagation of VLF and ELF Radio Waves When the Ionosphere Is Not Sharply Bounded ${ }^{1}$
}

\author{
James R. Wait \\ Contribution from Central Radio Propagation Laboratory, National Bureau of Standards, \\ Boulder, Colo.
}

(Received August 3, 1961)

\begin{abstract}
Employing an idea of Brekhovskikh, an expression for the reflection coefficient of a continuously stratified ionized medium is derived. The result is in the form of a series whose first term is a Fresnel-type coefficient and succeeding terms account for the finite thickness of the transition layer. This result is then fitted into previously developed theory for propagation between a spherical earth and a concentric ionosphere.
\end{abstract}

\section{Introduction}

In the mode theory of VLF radio propagation it is often assumed that the lower edge of the ionosphere may be represented by a sharply bounded and homogeneous ionized medium [Wait, 1960]. This assumption could be criticized as being unrealistic as it is known that the boundary between the non-ionized lower atmosphere and the ionosphere is "gradual." Unfortunately, the profiles of electron density and collision frequency, in the height range of importance for reflection of VLF waves at oblique incidence, are not well known. At these low heights (i.e., around $70 \mathrm{~km}$ ), the rocket measurements are not reliable and vertical incidence ionosondes give even less information. Some important qualitative data have come from the use of weak backscattered echoes at medium frequencies [Ratcliffe, 1960]. Among the findings of such work is the occurrence of very sharp gradients in electron density in the height regions of 60 to $80 \mathrm{~km}$.

In view of the preceding remarks, the sharply bounded model is not completely unjustified. Furthermore, it agrees with much of the experimental data at VLF if attention is restricted to highly oblique incidence. Nevertheless, it is desirable if more attention is paid to the possible influence of the "gradualness" of the ionosphere boundary. By making use of a digital computer some excellent progress has been made in this direction [Ferraro and Gibbons, 1959; Barron, 1959; Johler, 1962; Budden, 1961]. In particular, Johler [1961] has made a careful comparison between full wave solutions for certain gradual and sharply bounded models. His results indicate that the sharp boundary is often quite adequate.

It is the purpose of this paper to outline a relatively simple approach to the problem and to fit the results into the mode theory.

\section{Formulation}

To start with we shall assume an inhomogeneously stratified medium. With respect to a Cartesian coordinate system $(x, y, z)$, the refractive index $N(z)$ is assumed to be only a function of $z$. As $z$ tends to positive and negative infinity the refractive index is assumed to approach constant values. That is

and

$$
N(z)]_{z \rightarrow+\infty}=N_{1}
$$

$$
N(z)]_{z \rightarrow-\infty}=1 \text {. }
$$

At $z=-\infty$, a plane wave is incident at an angle $\theta_{0}$ with respect to the positive $z$ direction.

\footnotetext{
1 The research reported in this paper has been sponsored by the Advanced Research Projects Ageney, Washington 25, D.C.
} 
To simplify the discussion the earth's magnetic field is neglected. This is justified, at least in part, since the collision frequency is somewhat greater than the gyro frequency of electrons in the lowest ionosphere.

Thus

$$
N(z)=\left[1-i \frac{\omega_{r}(z)}{\omega}\right]^{\frac{1}{2}}
$$

where $\omega$ is the angular frequency, and $\omega_{r}(z)=\frac{\omega_{0}^{2}}{\nu+i \omega}$

where $\omega_{0}$ and $\mu$ are the (angular) plasma and collision frequencies, respectively [Ratcliffe, 1959]. These latter quantities are also functions of the coordinate $z$. It is convenient to introduce a dimensionless factor $L(z)$ in the following manner.

where

$$
\begin{gathered}
N(z)=\left(1-\frac{i}{L(z)}\right)^{\frac{1}{2}} \\
\frac{1}{L(z)}=\frac{\omega_{r}(z)}{\omega} \cong \frac{\omega_{0}^{2}}{(\nu+i \omega) \omega}
\end{gathered}
$$

Then

and

$$
L(z)]_{z \rightarrow+\infty}=L_{1}
$$

$$
L(z)]_{z \rightarrow-\infty}=\infty \text {. }
$$

In most applications at VLF, $\nu>>\omega$, so that $L(z)$ can be regarded as a real parameter.

The problem is to calculate the reflected wave at $z=-\infty$. If the electric vector of the incident wave was perpendicular to the $z$ axis, the resulting differential equations can be solved in terms of known functions provided certain profiles of $N(z)$ are used. The elegant analysis of P. S. Epstein [1930] for normal incidence can be carried over directly to this case (i.e., for horizontal polarization). Other closed-form solutions for the case of E-parallel polarization are also available in the literature [Brekhovskikh, 1960]. Unfortunately, the differentia] equations for $H$-parallel polarization (i.e., for vertical polarization) are somewhat more complicated and closed-form solutions for special transition profiles do not seem to be readily available. Thus a perturbation procedure is adopted following the suggestion of Brekhovskikh [1960].

\section{Differential Equation for the Reflection Coefficient}

Without any loss of generality, the $H$ vector is taken to have only a $y$ component. Thus, for a time factor, $e^{i \omega t}$, Maxwell's equations are given by

$$
\frac{\partial E_{x}}{\partial z}-\frac{\partial E_{z}}{\partial x}=-i \mu \omega H_{y}, \quad i \epsilon \omega E_{x}=-\frac{\partial H_{y}}{\partial z}, \text { and } i \epsilon \omega E_{z}=\frac{\partial H_{y}}{\partial x}
$$

where $\mu$ and $\epsilon$ are the magnetic permeability and permittivity of the medium. For sake of generality, both $\mu$ and $\epsilon$ can be regarded as functions of $z$. Of course, in applications to the ionosphere $\mu$ can be replaced by its free space value $\mu_{0}$. The retention of a variable $\mu$ in the theory permits one to readily adapt the results to arbitrary polarization. Furthermore, the analogy in acoustics is readily brought out.

Now the sum of the incident and reflected waves is defined in the following manner. The sole magnetic field component is written

$$
H_{y}=[A(z)+B(z)] \exp \left[-i(\epsilon \mu)^{\frac{1}{2}} S x\right]
$$

while the two electric field components are written

$$
E_{x}=[A(z)-B(z)] C(\mu / \epsilon)^{\frac{1}{2}} \exp \left[-i(\epsilon \mu)^{\frac{1}{2}} S x\right]
$$


and

$$
E_{z}=-[A(z)+B(z)] S(\mu / \epsilon)^{\frac{1}{2}} \exp \left[-i(\epsilon \mu)^{\frac{1}{2}} S x\right)
$$

in terms of $S$ and $C$ which can be regarded as sine and cosine of angle $\theta$ which is itself a function of $z$. At $z=-\infty$ the incident wave is taken to have an angle of incidence $\theta_{0}$.

In these equations, $k=\left(\epsilon_{0} \mu_{0}\right)^{\frac{1}{2}} \omega$ where $\epsilon_{0}$ and $\mu_{0}$ are the constants of free space, while $A(z)$ and $B(z)$ are not yet defined. Since these field components are to satisfy Maxwell's equations, it is required that

$$
(\epsilon \mu)^{\frac{1}{2}} S=\text { constant }
$$

and since $S=\sin \theta_{0}, \epsilon=\epsilon_{0}, \mu=\mu_{0}$, at $z=-\infty$, it follows that

$$
(\epsilon \mu)^{\frac{1}{2}} S=\left(\epsilon_{0} \mu_{0}\right)^{\frac{1}{2}} \sin \theta_{0}
$$

which is just a statement of Snell's law. Furthermore,

$$
C^{2}+S^{2}=1 \text {. }
$$

Again, as a consequence of Maxwell's equations, $A(z)$ and $B(z)$ must satisfy

$$
\begin{aligned}
& \frac{d A}{d z}+i \delta A+\Gamma(A-B)=0 \\
& \frac{d B}{d z}-i \delta B+\Gamma(B-A)=0
\end{aligned}
$$

where

and

$$
\delta=(\epsilon \mu)^{\frac{1}{2}} \omega C
$$

$$
\Gamma=\frac{\epsilon}{2 \delta} \frac{d}{d z}\left(\frac{\delta}{\epsilon}\right)
$$

Equations (8) and (9) are easily combined into a single equation for the ratio $B / A=R(z)$. Thus

$$
\frac{d R}{d z}=2 i \delta R+\Gamma\left(1-R^{2}\right)
$$

where $R(z)$ is, by definition, a reflection coefficient. Results, more or less equivalent to (10) have been given by Budden [1961] and Brekhovskikh [1960].

\section{Iterative Solution}

To obtain a solution, $R(z)$ is written in terms of a new function $v(z)$. Thus

$$
R=\frac{g(z) v(z)-g_{1}}{g(z) v(z)+g_{1}}
$$

where

$$
g(z)=\frac{K}{N^{2}}\left(N^{2}-S_{0}^{2}\right)^{\frac{1}{2}}, K=\mu / \mu_{0}, S_{0}=\sin \theta_{0}
$$

and

$$
g_{1}=\lim _{z \rightarrow \infty} g(z)=\frac{K_{1}}{N_{1}^{2}}\left(N_{1}^{2}-S^{2}\right), K_{1}=\mu_{1} / \mu_{0}
$$

Now, since $\lim _{z \rightarrow \infty} R(z)=0$, it follows that $\lim _{z \rightarrow \infty} v(z)=1$. The differential equation for $v(z)$ is obtained by substituting (11) into (10). This can be written in the relatively simple form

$$
-\frac{d v}{d z}=\frac{i k N^{2} g_{1}}{K}\left(1-\frac{g^{2}}{g_{1}} v^{2}\right)
$$

where $k=\left(\epsilon_{0} \mu_{0}\right)^{\frac{1}{2}} \omega$ and $N, K$, and $g$ are functions of $z$. Using a method of successive approximations (see for example, Z. Kopal [1955]), the solution can be expressed as an ascending 
series in powers of $k$. For example, the zeroth approximation is to replace the right-hand side of (12) by zero, thus $v$ is a constant which must be unity to satisfy the limiting condition at $z \rightarrow \infty$. The first approximation is obtained by replacing $v^{2}$ on the right-hand side by unity. Thus

$$
v=1+i k g_{1} \int_{z}^{\infty} \frac{N^{2}}{K}\left(1-\frac{g^{2}}{g_{1}}\right) d z
$$

where the limits of the integration are chosen so that $v$ satisfies the limiting condition at $z=\infty$. The second approximation is then obtained by substituting the latter result for $v$ into the right-hand side of (12). In general, the $n$th approximation, $v_{n}$, can be found from the $(n-1)$ th approximation, $v_{n-1}$, by using

$$
v_{n}=1+i k g_{1} \int_{z}^{\infty} \frac{N^{2}}{K}\left(1-\frac{g^{2}}{g_{1}^{2}} v_{n-1}^{2}\right) d z
$$

\section{Some Simple Extensions of the Solution}

While these results have been developed with specific reference to an incident wave with the electric vector in the plane of incidence, the results are also applicable to the other polarization. If the magnetic vector of the incident wave is in the plane of incidence (i.e., horizontal polarization), the results are still valid if the following transformations are made.

$$
H_{y} \rightarrow E_{y}, E_{x} \rightarrow-H_{x}, E_{z} \rightarrow-H_{z}, \mu \rightarrow \epsilon, \text { and } \epsilon \rightarrow \mu .
$$

Thus the formula for the reflection coefficient $R$, given by (11), is still valid if $K$ is replaced by $\epsilon / \epsilon_{0}$ and $N$ is not changed.

There is also a well-defined acoustic analogy to the problem being discussed. In this case $N(z)=\frac{c_{0}}{c(z)}$ where $c(z)$ is the velocity of sound and $c_{0}$ is the limiting value of $c$ at $z=-\infty$. Thus the velocity is varying from a constant value $c_{0}$ to a differing constant value $c_{1}$ at $z=+\infty$. Also, $K(z)=\rho(z) / \rho_{0}$ where $\rho(z)$ is the density and $\rho_{0}$ is its limiting value at $z=\infty$. The component $H_{y}$ is then analogous to the acoustic pressure and $E_{x}$ and $E_{z}$ are analogous to the $x$ and $z$ components, respectively, of the particle velocity.

\section{Discussion of the Form of the Solution}

It is interesting to note that for the zeroth approximation (corresponding to $v=1$ ), the reflection coefficient may be written

$$
R=\frac{C_{0}-\frac{K_{1}}{N_{1}^{2}}\left(N_{1}^{2}-S_{0}^{2}\right)^{\frac{1}{2}}}{C_{0}+\frac{K_{1}}{N_{1}^{2}}\left(N_{1}^{2}-S_{0}^{2}\right)^{\frac{1}{2}}} \text { where } C_{0}=\cos \theta_{0} \text { and } S_{0}=\sin \theta_{0} .
$$

This is the Fresnel reflection coefficient for the reflection of a plane wave at oblique incidence from a sharply bounded and homogeneous medium. Thus the higher terms in the ascending $k$ series account for the "gradualness" of the boundary.

In the general case, the reflection coefficient may be written

$$
R\left(z_{0}\right)=\frac{C_{0}-\left(K_{1} / N_{1}^{2}\right)\left(N_{1}^{2}-S_{0}^{2}\right)^{\frac{1}{2}} / v\left(z_{0}\right)}{C_{0}+\left(K_{1} / N_{1}^{2}\right)\left(N_{1}^{2}-S_{0}^{2}\right)^{\frac{1}{2}} / v\left(z_{0}\right)}
$$

where $z_{0}$ is some convenient level, below which $K(z)$ and $N(z)$ may be regarded as unity. Thus the total field in the region $z<z_{0}$ can be written

$$
H_{y}=H_{0} e^{-i k x S_{0}}\left[e^{-i k\left(z-z_{0}\right) C_{0}}+R\left(z_{0}\right) e^{+i k\left(z+z_{0}\right) C_{0}}\right]
$$


where $H_{0}$ is the value of the incident wave at the fictitious interface, $z=z_{0}$. In the second approximation, neglecting terms in $k^{3}$ and higher,

$$
v\left(z_{0}\right) \cong 1+i k g_{1} \int_{z_{0}}^{\infty} \frac{N^{2}}{K}\left(1-\frac{g^{2}}{g_{1}^{2}}\right) d z+2 k^{2} \int_{z_{0}}^{\infty} \frac{N^{2}}{K} g^{2}\left[\int_{z}^{\infty} \frac{N^{2}}{K}\left(1-\frac{g^{2}}{g_{1}^{2}}\right) d z\right] d z .
$$

Succeeding terms quickly become more complicated. It can be seen that the integrands contain the factor $1-\left(g / g_{1}\right)^{2}$ in each of these terms. The presence of this factor permits one to replace the upper limit of each of these integrals by $z_{1}$, where $z_{1}$ is the level above which $N(z)$ and $K(z)$ may be replaced by $N_{1}$ and $K_{1}$. Thus the transition region may be defined as the interval $z_{0}<z<z_{1}$. On this basis, it is apparent that the $n$th term in the series for $v\left(z_{0}\right)$ is the order of $\left[k\left(z_{1}-z_{0}\right)\right]^{n}$. Consequently, the series converges rapidly when the electrical thickness of the transition layer is small.

As an interesting check, the transition is replaced by a homogeneous slab. Thus,

$$
N(z)=\hat{N} \text { and } K(z)=\hat{K} \text { when } z_{0}<z<z_{1} .
$$

In this case, the reflection coefficient is given exactly by Wait [1958].

provided

$$
R=\frac{C_{0} v\left(z_{0}\right)-\left(N_{1}^{2}-S_{0}^{2}\right)^{\frac{1}{2}}\left(K_{1} / N_{1}^{2}\right)}{C_{0} v\left(z_{0}\right)+\left(N_{1}^{2}-S_{0}^{2}\right)^{\frac{1}{2}}\left(K_{1} / N_{1}^{2}\right)}
$$

$$
v\left(z_{0}\right)=\frac{1+\frac{K_{1} \hat{N}^{2}\left(N_{1}^{2}-S_{0}^{2}\right)^{\frac{1}{2}}}{\hat{K} N_{1}^{2}\left(\hat{N}^{2}-S_{0}^{2}\right)^{\frac{1}{2}}} \tanh \left[i k\left(z_{1}-z_{0}\right) \sqrt{\hat{N}^{2}-S_{0}^{2}}\right]}{1+\frac{\hat{K} N_{1}^{2}\left(\hat{N}^{2}-S_{0}^{2}\right)^{\frac{1}{2}}}{K_{1} \hat{N}^{2}\left(N_{1}^{2}-S_{0}^{2}\right)^{\frac{1}{2}}} \tanh \left[i k\left(z_{1}-z_{0}\right) \sqrt{\hat{N}^{2}-S_{0}^{2}}\right]} .
$$

If $\left|k\left(z_{1}-z_{0}\right) \sqrt{\hat{N}^{2}-S_{0}^{2}}\right|<<1$, it is seen that to a first order,

$$
v\left(z_{0}\right) \cong 1+\frac{\hat{N}^{2}\left(N_{1}^{2}-S_{0}^{2}\right)^{\frac{1}{2}}}{N_{1}^{2}}\left[1-\frac{\hat{K}^{2} N_{1}^{4}}{K_{1}^{2} \hat{N}^{4}} \frac{\hat{N}^{2}-S_{0}^{2}}{\hat{N}_{1}^{2}-S_{0}^{2}}\right] i k\left(z_{1}-z_{0}\right) \frac{K_{1}}{\hat{K}}
$$

which is consistent with the first two terms of the series given by (13).

For some applications to VLF propagation, it is desirable to express the reflection coefficient in the following form

where

$$
R=\frac{\beta C_{0}-1}{\beta C_{0}+1}
$$

$$
\beta=\frac{N_{1}^{2} v\left(z_{0}\right)}{K_{1}\left(N_{1}^{2}-S_{0}^{2}\right)^{\frac{2}{2}}}
$$

Then, if $\beta C_{0}$ is regarded as a small parameter, the following expansion results

$$
R=-e^{-2 \beta C_{0}}+\frac{2}{3}\left(\beta C_{0}\right)^{3}-\frac{4}{3}\left(\beta C_{0}\right)^{4}+\ldots
$$

Thus if $\left|\beta C_{0}\right|^{3} \ll 1$,

$$
R \cong-e^{-2 \beta C_{0}}
$$

which is a convenient form when the incidence is highly oblique and the frequency is not too low. On the other hand, if $\beta C_{0}$ is regarded as a large parameter, it is convenient to use the 
expansion

$$
R=\exp \left(-\frac{2}{\beta C_{0}}\right)-\frac{2}{3} \frac{1}{\left(\beta C_{0}\right)^{3}}+\frac{4}{3} \frac{1}{\left(\beta C_{0}\right)^{4}}+\ldots
$$

which can be approximated by the first term if $\left|\beta C_{0}\right|^{3} \gg 1$.

\section{Application to Terrestrial VLF Propagation}

The results developed in the above form may be readily fitted into VLF propagation theory which has been developed recently in a very general form [Wait, 1961]. The source of the field is a vertical electric dipole located on the surface of a smooth spherical earth of radius a, conductivity $\sigma$ and dielectric constant $\epsilon$. Spherical coordinates are chosen with the dipole located at $r=a$ and $\theta=0$. A concentric reflecting shell is now located at $r=a+h$. The electrical properties of the interfaces at $r=a$ and $r=a+h$ are defined in terms of two dimensionless factors, $q$ and $q_{i}$, respectively. These are defined explicitly below. Then, for harmonic time dependence, the electric field is written, apart from a constant factor, in the form [Wait, 1961]

where

$$
E_{r}=\frac{e^{-i k a \theta}}{a(\theta \sin \theta)^{\frac{1}{2}}} V
$$

$$
V=\sum_{j=0,1,2, \ldots} V_{j}
$$

The $V_{j}$ can be identified as sky waves or "hops", as they have a precise ray interpretation in the domain where geometrical optics is valid. Actually, in terms of the parameters of the problem [Wait, 1961],

for $j$ even, while

$$
V_{j}=e^{i \pi / 4}\left(\frac{x}{\pi}\right)^{\frac{1}{2}} \oint \frac{e^{-i x t} w_{1}(t-y)}{w_{1}^{\prime}(t)-q w_{1}(t)}[A(t) B(t)]^{j / 2} d t
$$

$$
V_{j}=e^{i \pi / 4}\left(\frac{x}{\pi}\right)^{\frac{1}{2}} \oint \frac{e^{-i x t} w_{2}(t-y)}{w_{1}^{\prime}(t)-q w_{1}(t)}[A(t)]^{\frac{j+1}{2}}[B(t)]^{\frac{j-1}{2}} d t
$$

for $j$ odd. In the above, $x=(k a / 2)^{1 / 3} \theta$ and $y=(2 / k a)^{1 / 3} k(r-a)$.

The contours of the integrations are to enclose all singularities of the integrands. The symbol $t$ has the following meaning

$$
(-t)^{\frac{1}{2}}=\left(\frac{k a}{2}\right)^{1 / 3} C
$$

where $C$ can be geometrically interpreted as the cosine of the (complex) angle of incidence on the earth's surface at $r=a$. Furthermore,

$$
\left(y_{0}-t\right)^{\frac{1}{2}}=\left(\frac{k a}{2}\right)^{1 / 3} C^{\prime}
$$

where $y_{0}=\left(\frac{2}{k a}\right)^{1 / 3} k h$ and $C^{\prime}$ can be geometrically interpreted as the cosine of the (complex) angle of incidence at the surface, $r=a+h$, which is regarded as the lower edge of the ionosphere. Other factors, found in (27), are

and

$$
A(t)=-\left[\frac{w_{1}^{\prime}\left(t-y_{0}\right)+q_{i} w_{1}\left(t-y_{0}\right)}{w_{2}^{\prime}\left(t-y_{0}\right)+q_{i} w_{2}\left(t-y_{0}\right)}\right]
$$

$$
B(t)=-\left[\frac{w_{2}^{\prime}(t)-q w_{2}(t)}{w_{1}^{\prime}(t)-q w_{1}(t)}\right]
$$

where $w_{1}(t)$ and $w_{2}(t)$ are Airy functions, defined elsewhere [Wait, 1961]. The factor $q$ occurring in the above is defined by 


$$
i q=\left(\frac{k a}{2}\right)^{1 / 3}\left(\frac{i \epsilon_{0} \omega}{\sigma+i \epsilon \omega}\right)^{\frac{1}{2}}\left[1-\frac{i \epsilon_{0} \omega}{\sigma+i \epsilon \omega}\right]^{\frac{1}{2}}
$$

since $\sigma>\rangle_{\omega} \omega$ in most cases of interest, this can be written

$$
q=\left(\frac{k a}{2}\right)^{1 / 3} G^{\frac{1}{2}} e^{-i \pi / 4}
$$

where

$$
G=\frac{\epsilon_{0} \omega}{\sigma} .
$$

To relate the factor $q_{i}$ to the properties of the ionosphere reflecting layer, a somewhat heuristic approach is used. First, it is assumed that $y_{0}-t$ has a real part somewhat greater than unity; thus, $w_{1}\left(t-y_{0}\right)$, etc., may be replaced by the first term of their asymptotic expansions. Then

$$
A(t) \cong \frac{C^{\prime}-\Delta_{i}}{C^{\prime}+\Delta_{i}} \exp \left[-i \frac{\pi}{2}-i \frac{4}{3}\left(\frac{k a}{2}\right)\left(C^{\prime}\right)^{3}\right]
$$

where $\Delta_{i}=\left(\frac{2}{k a}\right)^{1 / 3} i q_{i}, \quad$ As indicated in a previous analysis [Wait, 1961], $\left(C^{\prime}-\Delta_{i}\right) /\left(C^{\prime}+\Delta_{i}\right)$ is a reflection coefficient referred to the bottom of the layer $r=a+h$. Therefore, $\Delta_{i}$ may be identified with the factor $1 / \beta$; this is certainly evident on comparing (22a) and (34). Implicit in the above identification is that the local reflection process at the ionosphere does not depend on earth curvature. It should be emphasized that, in general, $q_{i}$ and $\beta$ are functions of $C^{\prime}$ and thus, in turn, they are functions of $t$, the variable of integration. However, for propagation to great distances, $q_{i}$ and $\beta$ may be replaced by a (complex) constant appropriate for grazing incidence (i.e., $S_{0} \rightarrow 1$ ).

On utilizing $(2),(21)$, and $(22 \mathrm{~b})$, it is seen that to a first order in $k$,

$$
\beta \cong\left(\sqrt{L_{1}}-\frac{i}{\sqrt{L_{1}}}\right) \sqrt{i}+i k \int_{z_{0}}^{z_{1}} \frac{1}{\left(1-\frac{i}{L}\right)}\left[\left(1-\frac{1}{L^{2}}\right)-\left(1-\frac{1}{L_{1}^{2}}\right) \frac{L_{1}}{L}\right] d z
$$

or

$$
\beta \simeq\left(\sqrt{L_{1}}-\frac{i}{\sqrt{L_{1}}}\right) \sqrt{i}+k \int_{z_{0}}^{z_{1}} \frac{i-\frac{1}{L}}{1+\frac{1}{L^{2}}}\left[\left(1-\frac{L_{1}}{L}\right)\left(1+\frac{1}{L L_{1}}\right)\right] d z .
$$

Then, $i q_{i}=(k a / 2)^{1 / 3}(1 / \beta)$. In the above, it is understood that $L$ is a function of $z$ in the transition region extending from $z_{0}$ to $z$. If $k\left(z_{1}-z_{0}\right)$ is not small compared with unity, it is necessary to obtain higher order terms.

As has been demonstrated before [Wait, 1961], the waveguide modes are found from the equation

$$
A(t) B(t)=e^{-i 2 \pi n}
$$

where $n$ is an integer. Some of the methods developed for solving this equation in the sharply bounded limit (i. e., $z_{1} \rightarrow z_{0}$ ) are also applicable to the "gradual" model.

\section{Application to ELF Propagation}

At ELF (extremely low frequencies), the influence of the finite transition region can be quite simply indicated. In this case, the modal equation can be written

where

$$
R_{g}(C) R_{i}(C) e^{-i 2 k h C}=e^{-i 2 \pi n}
$$

and

$$
R_{g}(C) \cong \exp [-(2 / C) \sqrt{i G}]
$$

$$
R_{i}(C) \cong \exp [-(2 / C)(1 / \beta)] .
$$


These are valid subject to the restrictions that $|C|>>\left(\frac{h}{a}\right)^{\frac{1}{2}},\left(\frac{2}{k a}\right)^{\frac{1}{3}},|1 / \beta|$, and $\sqrt{G}$. Equation (38) may be written

$$
k h C=\pi n+i \frac{\Delta}{C} \text { where } \Delta=\sqrt{i G}+1 / \beta .
$$

This is solved as a quadratic to yield

$$
2 C_{n}=\frac{\pi n}{k h} \pm\left[\left(\frac{\pi n}{k h}\right)^{2}+4 i \frac{\Delta}{k h}\right]^{\frac{1}{2}}
$$

The positive sign before the radical is chosen since it reduces to $C_{n}=(\pi n / k h)$ when $\Delta$ approaches zero. The corresponding values of $t$ are then found from

$$
\left(-t_{n}\right)^{\frac{1}{2}}=(k a / 2)^{\frac{1}{3}} C_{n} \text {. }
$$

Individual modes propagate in the horizontal direction according to $(\sin \theta)^{-\frac{1}{2}} \exp \left(-i x t_{n}\right)$ $\exp (-i k a \theta)$ which is equivalent to

$$
[\sin (d / a)]^{-\frac{1}{2}} \exp \left(-i k d S_{n}\right), \text { with } d=a \theta,
$$

where $S_{n}=\left(1-C_{n}^{2}\right)^{\frac{1}{2}}$. Since $|\Delta| k h<<1$, the radical in (41) may be expanded to yield

and

$$
S_{n}=\left[1-\left(\frac{\pi n}{k h}\right)^{2}-i \frac{2 \Delta}{k h}\right]^{\frac{1}{2}} \text { for } n=1,2,3 \ldots
$$

$$
S_{0}=\left[1-i \frac{\Delta}{k h}\right]^{\frac{1}{2}} \text {. }
$$

Usually the zero-order mode is of greatest interest since the higher modes are beyond "cut-off." Furthermore, in most cases $|\Delta|<<k h$; therefore, the propagation is adequately described by the zero-order mode which may be expressed in the form

$$
S_{0} \cong 1-\frac{i \Delta}{2 k h}=1-\frac{i}{2 k h}\left[\frac{1}{\beta}+\sqrt{G} e^{i \pi / 4}\right]
$$

The attenuation in nepers per unit distance is thus given by

where

$$
-k \operatorname{Im} S_{0} \cong \frac{1}{2 h}\left[\operatorname{Re} \frac{1}{\beta}+\sqrt{G / 2}\right]
$$

$$
\operatorname{Re} \frac{1}{\beta} \cong \sqrt{L_{1} / 2}+k L_{1} \int_{h}^{h+b} \frac{\left(1-\frac{L_{1}}{L}\right)\left(1+\frac{1}{L L_{1}}\right)}{\left(1+\frac{1}{L^{2}}\right)} d z
$$

When $b$ tends to zero, corresponding to a sharp boundary, the integral term vanishes and the attenuation factor is given by

$$
-k \operatorname{Im} S_{0} \cong \frac{1}{2 \sqrt{2 h}}\left[\sqrt{L_{1}}+\sqrt{G}\right]
$$

which is well-known. When the dependence of $L(z)$ with height is specified it is a relatively simple matter to calculate the correction resulting from the "gradualness" of the boundary. In general it can be seen that, for the ELF range, the contribution from the second integral is always positive since $L>>L_{1}$ over the transition region. Therefore, the presence of the transition layer is to increase the attenuation relative to that of a sharply bounded model.

Specific numerical results for mode characteristics based on this approach will be given in a later paper. In particular, it is hoped to make use of the extensive numerical results of Johler [1961, 1962] for the plane wave reflection coefficients. 


\section{References}

Barron, D. W., The 'waveguide mode' theory of radio wave propagation when the ionosphere is not sharply bounded, Phil. Mag. 45, No. 4, 1068-1081 (Sept. 1959).

Brekhovskikh, L. M., Waves in layered media (Academic Press, London and New York, 1960).

Budden, K. G., Radio waves in the ionosphere (Cambridge University Press, 1961).

Epstein, P. S., Reflection of waves in an inhomogeneous absorbing medium, Proc. Nat. Acad. Sci. (U.S.A.) 16, $627(1930)$

Ferraro, A. J., and J. J. Gibbons, Polarization computations by means of the multi-slab approximation, J. Atmospheric and Terrest. Phys. 16, 136-144 (1959).

Johler, J. R., On the analysis of LF ionospheric radio propagation phenomena, J. Research NBS 65D (Radio Prop.), No. 5, 507 (Sept.-Oct. 1961)

Johler, J. R., and J. D. Harper, Jr., Reflection and transmission at a continuously stratified plasma with arbitrary magnetic induction, J. Research NBS 66D (Radio Prop.), No. 1 (Jan.-Feb. 1962).

Kopal, Z., Numerical analysis, p. 145 (John Wiley \& Sons, Inc., New York, N.Y., 1955).

Ratcliffe, J. A., Magneto-ionic theory (Cambridge University Press, 1959).

Ratcliffe, J. A. (editor), Physics of the upper atmosphere, p. 404 (Academic Press, London and New York, 1960).

Wait, J. R., An extension to the mode theory of VLF ionospheric propagation, J. Geophys. Research 63, No. 1, 125-135 (Mar. 1958).

Wait, J. R., Terrestrial propagation of VLF radio waves, J. Research NBS 64D (Radio Prop.), No. 2, 153-204 (Mar--Apr. 1960). (Many references are given here.)

Wait, J. R., A new approach to the mode theory of VLF propagation, J. Research NBS 65D (Radio Prop.), No. $1,37-46$ (Jan.-Feb. 1961).

(Paper 66D1-173) 